\title{
Pharmacokinetic Comparison of Isoalantolactone and Alantolactone in Rats after Administration Separately by Optimization of an UPLC-MS ${ }^{2}$ Method
}

\author{
Renjie Xu, Mengyue Wang, Ying Peng, and Xiaobo Li \\ School of Pharmacy, Shanghai Jiao Tong University, No. 800 Dongchuan Road, Minhang District, Shanghai 200240, China \\ Correspondence should be addressed to Xiaobo Li; xbli@sjtu.edu.cn
}

Received 2 September 2014; Accepted 4 November 2014; Published 20 November 2014

Academic Editor: Patricia Valentao

Copyright (C) 2014 Renjie Xu et al. This is an open access article distributed under the Creative Commons Attribution License, which permits unrestricted use, distribution, and reproduction in any medium, provided the original work is properly cited.

\begin{abstract}
Isoalantolactone and alantolactone are two major active ingredients that are present in many medicinal plants. In this study, a sensitive and rapid ultraperformance liquid chromatography tandem mass spectrometry (UPLC-MS/MS) method was developed for determination of the two compounds in rat plasma, separately. In this method, an electrospray ionization source was applied and operated in positive ion mode; multiple reaction monitoring (MRM) was selected for quantification using target fragment ions $233.2 \rightarrow 187.1$ for isoalantolactone (alantolactone) and $245.1 \rightarrow 189.1$ for internal standard (IS). Retention time of the lactones and IS was within $3.0 \mathrm{~min}$. Further calibration suggested a linear regression can be calculated within $2.5-500 \mathrm{ng} / \mathrm{mL}$ for isoalantolactone and $4-500 \mathrm{ng} / \mathrm{mL}$ for alantolactone. This method was used to compare the pharmacokinetic characteristics of isoalantolactone and alantolactone at a single dose of $5 \mathrm{mg} / \mathrm{kg}$ into male Sprague-Dawley rats by intravenous administration separately. The levels of $t_{1 / 2}, \mathrm{Kel}, \mathrm{CL}, C_{\max }$, and AUC were significantly increased in the alantolactone group compared to isoalantolactone. These results suggested that isoalantolactone was distributed and eliminated more rapidly than alantolactone in rats when administered, respectively.
\end{abstract}

\section{Introduction}

Isoalantolactone and alantolactone were first isolated from Inula helenium [1] and have been found in many Inula plants such as I. royleana [2] and I. japonica [3]; also they have been isolated from Aucklandia lappa [4] and Chinese medicine preparation called Liuwei Anxiao San [5]. Isoalantolactone and alantolactone have a wide range of activities both in vivo and in vitro, including antimicrobial $[6,7]$, anti-inflammatory, and antitrypanosomal activities [8], and protect against Staphylococcus aureus pneumonia [9]. Moreover, isoalantolactone was reported to reduce significantly the mitochondrial potential $(\Delta \Psi \mathrm{m})$ in pancreatic carcinoma PANC-1 cells [10]. The effects of alantolactone on the intrinsic pathway of apoptosis have been examined in many cell lines, including human leukemia K562 [11] and glioblastoma U87 cells [12]. These studies suggest that alantolactone and isoalantolactone are potential promising anticancer candidates but in various applications. First of all is to develop a method for determination of isoalantolactone and alantolactone in plasma in preclinical studies and subsequent investigations. However, despite being critical preclinical parameter [13], the pharmacokinetics of these compounds were rarely studied to this date and we found the concentrations of isoalantolactone and alantolactone were too low to be determined by UV scan after intravenous injection due to the end of absorption of the lactones $(210 \mathrm{~nm})$. LC coupled with tandem mass spectrometer (LCMS/MS) techniques frequently provided specific, selective, and sensitive quantitative results and reduced sample preparation and analysis time compared with the other common used techniques [14].

A simultaneous determination of isoalantolactone and alantolactone in rat plasma via intravenous injection was reported in a recent study [15], and it turned out that the pharmacokinetic parameters of the two lactones were analogous. Unfortunately, alantolactone was found to induce detoxifying enzymes such as quinone reductase $(\mathrm{QR})$ and 


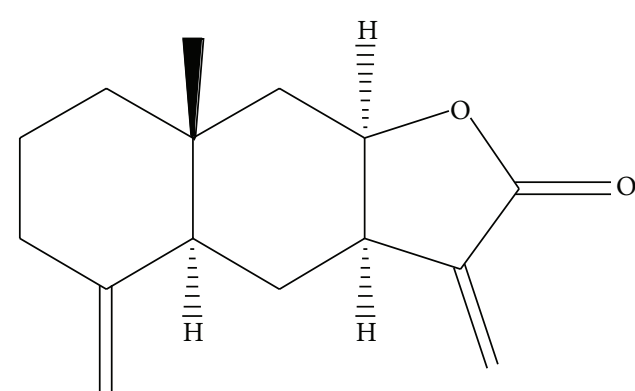

(a)

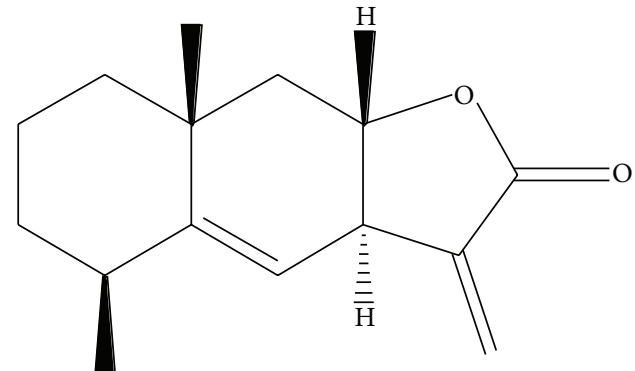

(b)

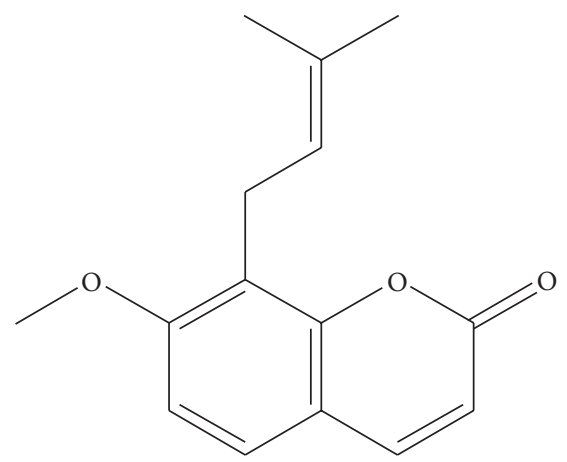

(c)

FIGURE 1: Structures of isoalantolactone (a), alantolactone (b), and osthole ((c), IS).

glutathione S-transferase (GST) activity [16], suggesting that the pharmacokinetic parameters by simultaneous administration might not be accurate because of the drug interactions in metabolism in vivo. Furthermore, total analysis time of this method is too long by HPLC for almost $19 \mathrm{~min}$ and the retention time of IS and lactones was so different $(6.4 \mathrm{~min}$ versus $12.4 \mathrm{~min}$ ).

In present study, we developed a novel customized UPLCMS/MS method to determine isoalantolactone and alantolactone in rat plasma with a shorter retention time (less than $3 \mathrm{~min}$ ) and lower LLOQ by optimized MS conditions. Osthole was selected as the internal standard (IS) since its retention time was similar to the two lactones. This method was validated and applied to the pharmacokinetic studies of isoalantolactone and alantolactone separately via intravenous injection in rats models with a longer sample collection time than the study before [15] (10 h to $50 \mathrm{~min}$ ) which made the pharmacokinetic parameters more accurate. At the end, we found the pharmacokinetic parameters of isoalantolactone were so different from those of alantolactone after administration separately.

\section{Experimental}

2.1. Chemicals and Reagents. Osthole (purity > 98.0\%, Figure 1(c)) as internal standard (IS) was obtained from the National Institute for the Control of Pharmaceutical and Biological Products (Beijing, China). HPLC-grade acetonitrile was purchased from Merck Company (Darmstadt, Germany). Ultrapure water for the LC mobile phase was prepared in-house using a Milli-Q system (Millipore, Bedford, MA,
USA). All other chemicals were of analytical grade and were used without further purification.

2.2. Instrumentation and Analytical Conditions. All analysis was performed with The UPLC-MS/MS system that consisted of a Waters UPLC system with an Applied Biosystem 5500 QTRAP hybrid triple-quadrupole mass spectrometer (Applied Biosystems/MDS Sciex, Foster City, CA, USA), equipped with a turbo ion spray source. Chromatographic separation was performed on a ZORBAX Eclipse Plus $\mathrm{C}_{18}$ $(50 \mathrm{~mm} \times 2.1 \mathrm{~mm}, 1.8 \mu \mathrm{m})$. The mobile phase consisted of (A) deionized water and (B) acetonitrile, and the flow rate was $0.4 \mathrm{~mL} / \mathrm{min}$. The following gradient elutions were used: 0.0-3.0 min, $30.0 \rightarrow 74.0 \% \mathrm{~B} ; 3.0-3.1 \mathrm{~min}, 74.0 \rightarrow 95.0 \% \mathrm{~B}$; 3.1-4.6 $\min 95 \% \mathrm{~B}$; $4.6-4.7 \mathrm{~min}, 95.0 \rightarrow 30 \% \mathrm{~B}$; and $4.7-$ $7.0 \mathrm{~min}, 30 \% \mathrm{~B}$. The column was maintained at $50^{\circ} \mathrm{C}$ and the autosampler tray at $20^{\circ} \mathrm{C}$.

Nitrogen was used as the nebulizer (GS1), heater (GS2), curtain (CUR) gas, and the collision activation dissociation (CAD) gas. Tandem MS analyses were performed in the MRM acquisition mode for simultaneous detection of all the targeted analytes, with two precursor-to-product ion transitions monitored for the two compounds. The source settings and instrument parameters for each MRM transition were optimized to maximize the generation of protonated analyte molecule $\left([\mathrm{M}+\mathrm{H}]^{+}\right)$of each targeted compound, as well as efficiently produce its characteristic fragments/product ions. The ion spray needle voltage was set at $5500 \mathrm{~V}$ and the source temperature was $550^{\circ} \mathrm{C}$. Following optimization of the setting parameters, the ESI source was operated with the CUR, GS1, and GS2 set at 40, 35, and 35 psi, respectively. 
The compound-dependent instrumental parameters of two individual precursor-to-product ion transitions specific for each analyte including precursor ion, two product ions, declustering potential (DP), entrance potential (EP), collision energy (CE), and collision cell exit potentials (CXP) were optimized. The parameters are $50 \mathrm{~V}, 19 \mathrm{eV}, 10 \mathrm{~V}$, and 12 successively for both lactones and IS. The transition from precursor ion $[\mathrm{M}+\mathrm{H}]^{+}$at $m / z 233.2$ to product ion at $\mathrm{m} / z$ 187.1 was monitored; to detect osthole, the transition from the precursor at $m / z 245.1$ to the product at $m / z 189.1$ was monitored. The dwell time was set at $550 \mathrm{~ms}$.

\subsection{Preparation of Isoalantolactone and Alantolactone. Inula} helenium $(1 \mathrm{~kg})$ was refluxed two times with $95 \%$ ethanol at $100^{\circ} \mathrm{C}$ for $2 \mathrm{~h}$. The extraction solutions were combined, and ethanol was removed under reduced pressure. The residue was suspended in water and extracted with chloroform. The resulting solution was evaporated under reduced pressure and offered a brown residue that contained isoalantolactone and alantolactone. The two lactones were separated by repeated silica column chromatography (contained $10 \%$ $\left.\mathrm{AgNO}_{3}\right)$ and eluted with petroleum ether and acetone $(100: 2)$. Isoalantolactone $(48 \mathrm{mg})$ and alantolactone $(36 \mathrm{mg})$ were collected, respectively (Figures 1(a) and 1(b)).

2.4. Preparation of Stock and Working Solutions. Primary stock solutions of two lactones for preparation of standard and quality control (QC) samples were prepared from separate weighing. Stock solutions $(1.0 \mathrm{mg} / \mathrm{mL})$ of both lactones and IS were prepared in acetonitrile. The isoalantolactone (alantolactone) stock solution was diluted with acetonitrile to generate working solutions with concentrations ranging from $25 \mathrm{ng} / \mathrm{mL}$ to $5000 \mathrm{ng} / \mathrm{mL}$ (concentrations $40,50,100$, $200,500,1000$, and $5000 \mathrm{ng} / \mathrm{mL}$ ) and alantolactone from $40 \mathrm{ng} / \mathrm{mL}$ to $5000 \mathrm{ng} / \mathrm{mL}$ (concentrations $25,50,100,200$, 500,1000 , and $5000 \mathrm{ng} / \mathrm{mL}$ ) for calibration curves; IS working solutions were prepared with acetonitrile at $5 \mathrm{ng} / \mathrm{mL}$.

2.5. Calibration Standards and Quality Control Samples. Calibration samples were prepared by spiking $90 \mu \mathrm{L}$ of control rat plasma with the appropriate $10 \mu \mathrm{L}$ of working solutions of the analytes on the day of analysis. All solutions were stored at $4^{\circ} \mathrm{C}$ and brought to room temperature before use. Quality control (QC) samples were prepared in the same way as the calibration standards, with three plasma concentrations at 5 , 50 , and $400 \mathrm{ng} / \mathrm{mL}$. The analytical standards and QC samples were stored at $-80^{\circ} \mathrm{C}$.

2.6. Sample Preparation. Before analysis, the plasma samples were thawed to room temperature. In a $1.5 \mathrm{~mL}$ centrifuge tube, an aliquot of $10 \mu \mathrm{L}$ of the internal standard working solution $(5 \mathrm{ng} / \mathrm{mL})$ was added to $100 \mu \mathrm{L}$ of collected plasma sample followed by the supplementary addition of $390 \mu \mathrm{L}$ of acetonitrile $(4: 1, \mathrm{v} / \mathrm{v})$. The tubes were vortex-mixed for $1 \mathrm{~min}$ and centrifuged at $14,000 \mathrm{rpm}$ for $10 \mathrm{~min}$. The supernatant $(2 \mu \mathrm{L})$ was injected into the UPLC-MS/MS system for analysis directly.
2.7. Method Validation. The method was validated for selectivity, linearity, accuracy, precision, recovery, and stability according to the literatures for validation of bioanalytical methods (Food and Drug Administration 2001). Validations were conducted on three consecutive days. Each validation consisted of six replicates of QC plasma samples.

2.7.1. Specificity and Selectivity. The selectivity of the method was evaluated by analyzing blank rat plasma and blank plasma spiked with lactones and IS as well as a rat plasma sample. The selectivity and specificity validations were carried out by analyzing six different blank plasma samples from rats to demonstrate the lack of chromatographic interference from endogenous plasma at the retention time of the analytes and IS.

2.7.2. $L O D, L O Q$, and Linearity. The lower limit of determination (LOD) had to fulfill the requirement that the signal-tonoise ratio was at least $3: 1$ and the accuracy did not deviate by more than $20 \%$. The lower limit of quantification (LLOQ), defined as the lowest concentration on the calibration curve, had to fulfill the requirement that the signal-to-noise ratio was at least 10:1 and the accuracy did not deviate by more than $20 \%$. Calibration curves were constructed by assaying calibration standards spiked with seven nominal lactones concentrations as in Section 2.5 mentioned and then processed as in Section 2.6.

2.7.3. Precision and Accuracy. The precision of the method was determined by calculating RSD for QCs at three concentration levels over three validation days. The accuracy of the method was determined by calculating RE at each QC level.

2.7.4. Recovery and Matrix Effect. By determining the absolute peak areas of lactones at all the three QC levels in six samples, the matrix effect and recovery of the method were evaluated, including standards, standards spiked prior to the extraction, and standards spiked after extraction in six samples. Matrix effect was calculated by dividing the peak area of isoalantolactone and alantolactone in samples spiked postextraction by that of the acetonitrile dissolved samples. The extraction recovery was calculated by dividing the peak area of standards spiked prior to the extraction by that of the isoalantolactone and alantolactone spiked postextraction sample.

2.7.5. Stability. The stability of QC samples was investigated at 5,50 , and $400 \mathrm{ng} / \mathrm{mL}$. The stability investigation included stability in conditions after three freeze-thaw cycles, room temperature for $4 \mathrm{~h}$, autosampler stability of analysis in the room temperature for $24 \mathrm{~h}$, and stability in the stock solution for 20 days at $-80^{\circ} \mathrm{C}$.

2.7.6. Application of the Method to Pharmacokinetic Study. An ideal chromatographic separation was achieved on a reversed phase Zorbax Eclipse Plus- $\mathrm{C}_{18}(2.1 \times 50 \mathrm{~mm}, 1.8 \mu \mathrm{m})$ column within $7 \mathrm{~min}$. An electrospray ionization source was applied and operated in positive ion mode; MRM was used 


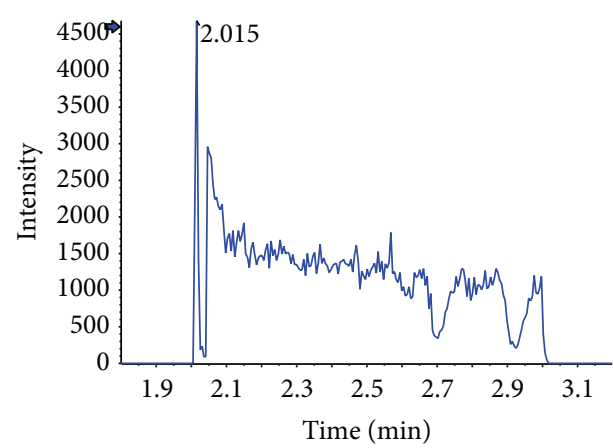

(a)
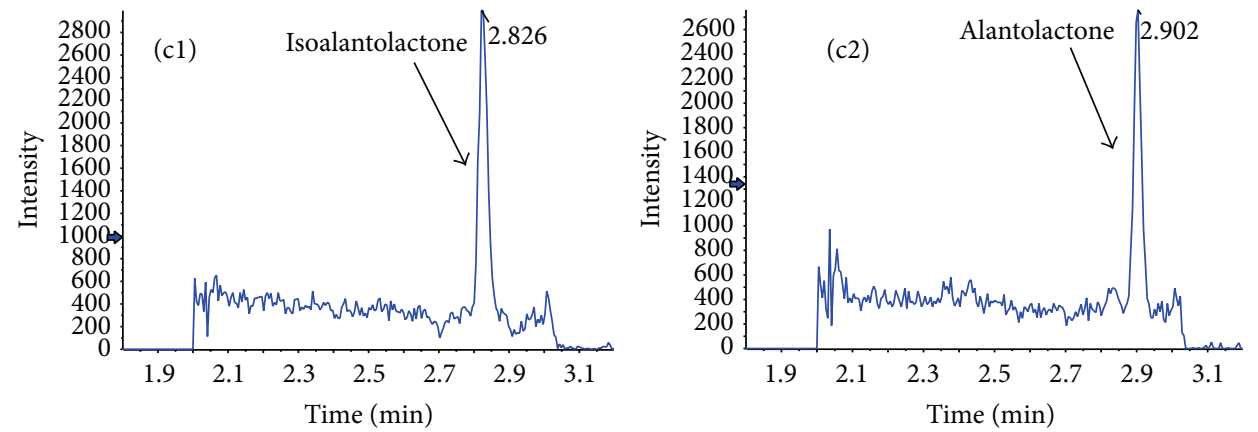

(c)
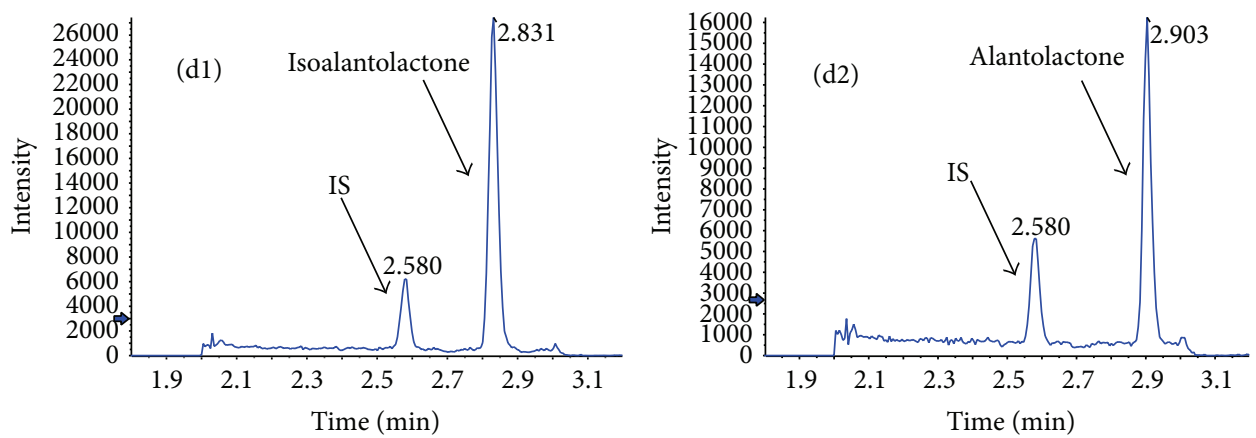

(d)
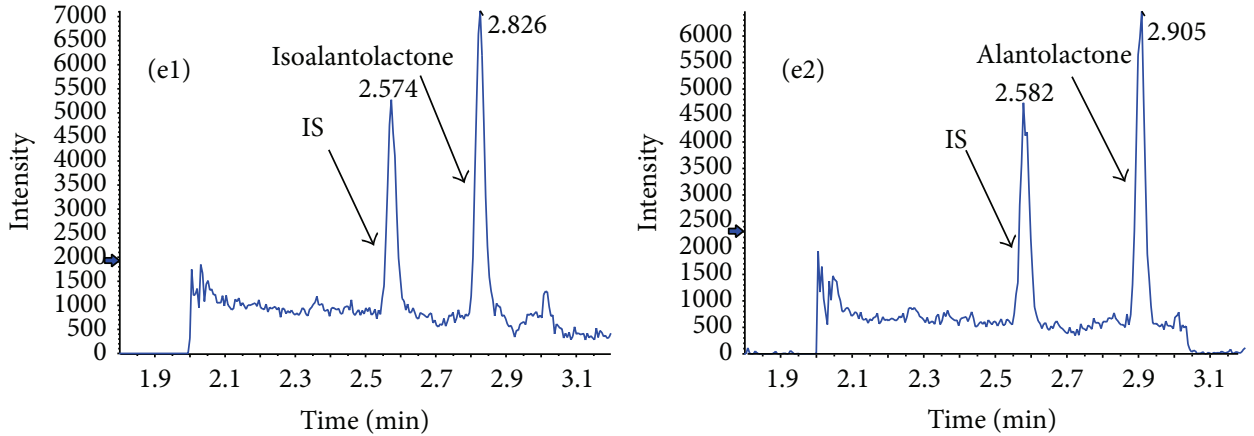

(e)

FIGURE 2: Representative multiple reaction monitoring chromatograms of (a) a blank plasma sample of rats, (b) a blank rat plasma sample spiked with IS $(0.1 \mathrm{ng} / \mathrm{mL})$, (c) a blank rat plasma sample spiked with lactones ((c1) for isoalantolactone at $2.5 \mathrm{ng} / \mathrm{mL}$ and (c2) for alantolactone at $4 \mathrm{ng} / \mathrm{mL}),(\mathrm{d})$ acetonitrile spiked with IS and lactones ((d1) for isoalantolactone and (d2) for alantolactone), and (e) a rat plasma sample obtained $2 \mathrm{~h}$ after intravenous injection of $5 \mathrm{mg} / \mathrm{kg}$ isoalantolactone (e1) and $4 \mathrm{~h}$ of alantolactone (e2). 
for quantification using target fragment ions $233.2 \rightarrow 187.1$ for isoalantolactone (alantolactone) and $245.1 \rightarrow 189.1$ for the osthole. Twelve male Sprague-Dawley rats (200-220 g) about 6 weeks were obtained from the Laboratory Animal Centre of Shanghai Jiao Tong University (Shanghai, China). Before the experiments, rats were housed in a temperature $\left(25 \pm 2^{\circ} \mathrm{C}\right)$ and moisture $(55 \pm 10 \%)$ controlled room, exposed to a controlled $12 \mathrm{~h}$ light/dark cycle, and allowed unrestricted access to food and water ad libitum. The animal experimentation was approved by the Shanghai Jiao Tong University Animal Ethics Committee (Shanghai, China).

Accurately weighed isoalantolactone was suspended in 5\% PEG-400 deionized water and the same operation for alantolactone. For the pharmacokinetic study, isoalantolactone and alantolactone at $5 \mathrm{mg} / \mathrm{kg}$ were injected intravenously into the tail vein in six rats separately. Blood samples were collected from the orbital sinus before and after dosing between 0.083 and $10 \mathrm{~h}$ and immediately centrifuged at $5000 \mathrm{rpm}$ for $10 \mathrm{~min}$ to obtain plasma samples, which were stored at $-80^{\circ} \mathrm{C}$ until LC-MS/MS analysis.

Drug and Statistics Software Kenitica 4.4.1 was used to analyze plasma isoalantolactone concentration over time in each experimental rat. Mass processing software version was MultiQuant 2.1.1.

\section{Investigations Results and Discussion}

3.1. Optimization of Mass Spectroscopy Conditions. Quadrupole full scans were carried out in both positive and negative ion detection mode using a $100 \mathrm{ng} / \mathrm{mL}$ tuning solution in methanol. A good response was achieved in positive ionization mode for isoalantolactone, alantolactone, and IS. The protonated molecular ions for isoalantolactone, alantolactone, and IS revealed peaks at $m / z$ 233.2, 233.2, and 245.1, respectively, and $[\mathrm{M}+\mathrm{H}]^{+}$ions were found using a direct infusion experiment. The most abundant ions produced by the target protonated molecular ions are 187.1 for the two isomers. A similar situation occurred during the analysis of IS as previously reported [17].

The mobile phase, as well as types of chromatography columns and column temperatures, was tested to optimize the chromatographic conditions. An acetonitrile-water mobile phase system from $30 \%$ to $95 \%$ acetonitrile without any acid in $3.10 \mathrm{~min}$ was proven to strengthen the mass spectrum response of isoalantolactone and alantolactone. Zorbax Eclipse Plus- $\mathrm{C}_{18}(2.1 \times 50 \mathrm{~mm}, 1.8 \mu \mathrm{m})$ analytical column was selected to give the most satisfactory chromatography from other commercially available $\mathrm{C}_{18}$ columns such as Poroshell $120 \mathrm{EC}-\mathrm{C}_{18}(3.0 \times 50 \mathrm{~mm}, 2.7 \mu \mathrm{m})$, Zorbax Eclipse Plus- $\mathrm{C}_{18}$ $(2.1 \times 50 \mathrm{~mm}, 1.8 \mu \mathrm{m})$, and Acquity UPLC BEH C 18 column $(2.1 \times 50 \mathrm{~mm}, 1.7 \mu \mathrm{m})$. Three column temperatures $\left(30^{\circ} \mathrm{C}\right.$, $40^{\circ} \mathrm{C}$, and $50^{\circ} \mathrm{C}$ ) were tested and $50^{\circ} \mathrm{C}$ was selected due to the shortest retention time. Based on the above analysis, isoalantolactone and alantolactone were eluted at retention time 2.83 and $2.90 \mathrm{~min}$ and IS was at $2.60 \mathrm{~min}$. UPLCMS/MS was performed in MRM mode with target ions at $m / z$ $233.2 \rightarrow 187.1$ for isoalantolactone (alantolactone) and $\mathrm{m} / \mathrm{z}$ $245.1 \rightarrow 189.1$ for the osthole.
TABLE 1: Precision and accuracy of QC samples in rat plasma $(n=6)$.

\begin{tabular}{lcccc}
\hline Concentration & \multicolumn{2}{c}{ RSD (\%) } & \multicolumn{2}{c}{ RE (\%) } \\
$(\mathrm{ng} / \mathrm{mL})$ & Intraday & Interday & Intraday & Interday \\
\hline Isoalantolactone & & & & \\
$\quad 5$ & 3.73 & 5.24 & -12.57 & -9.83 \\
50 & 2.41 & 3.59 & -10.86 & -9.23 \\
400 & 3.19 & 5.64 & -4.20 & 6.62 \\
Alantolactone & & & & \\
5 & 4.25 & 9.57 & 10.90 & 14.53 \\
50 & 6.23 & 9.33 & 9.18 & -9.22 \\
400 & 7.06 & 7.56 & 2.78 & -6.13 \\
\hline
\end{tabular}

TABLE 2: Extraction recovery and matrix effect of the method $(n=$ $6)$.

Nominal concentration Extraction recovery Mean matrix effect (ng/mL)

(\%)

(\%)

\begin{tabular}{lcc}
\hline Isoalantolactone & & \\
5 & $93.59 \pm 5.56$ & $109.11 \pm 6.69$ \\
50 & $89.33 \pm 4.06$ & $108.32 \pm 3.76$ \\
$\quad 400$ & $84.02 \pm 2.05$ & $103.54 \pm 3.98$ \\
Alantolactone & & \\
5 & $91.91 \pm 6.01$ & $109.44 \pm 2.00$ \\
50 & $88.64 \pm 4.53$ & $107.91 \pm 3.85$ \\
$\quad 400$ & $83.61 \pm 2.52$ & $100.84 \pm 2.88$ \\
IS & & \\
$\quad 0.1$ & $94.09 \pm 8.61$ & $101.76 \pm 5.96$ \\
\hline
\end{tabular}

3.2. Sample Pretreatment Method. To minimize potential interferences of rat plasma matrix to UPLC-MS/MS system, a simple, rapid protein precipitation pretreatment was performed as previously reported [18]. Over $99.7 \%$ of proteins in plasma samples were precipitated using four acetonitrile volumes. The solid phase extraction column $\mathrm{C}_{18}$ was also tried. The samples were purified by water and eluted with $100 \%$ methanol, and then the eluent was dried, reconstituted in $100 \mu \mathrm{L}$ of mobile phase, vortex-mixed for $30 \mathrm{~s}$, and centrifuged at $14,000 \mathrm{rpm}$ for $5 \mathrm{~min}$. Finally, $2 \mu \mathrm{L}$ of supernatant was injected into the UPLC-MS/MS system for analysis. It was found to be inapplicable due to large standard deviation and the cumbersome steps.

\subsection{Method Validation}

3.3.1. Specificity and Selectivity. Both lactones and IS were well extracted and separated from the blank matrix as shown in Figure 2. All the peaks of the analytes and IS were detected with excellent resolution as well as peak shapes. Both lactones and IS were eluted within $3.10 \mathrm{~min}$, with retention time for lactones and IS between $2.63 \mathrm{~min}$ and $3.10 \mathrm{~min}$. There was no cross-reaction from plasma in the chromatogram.

3.3.2. LOD, LOQ, and Linearity. The limit of detection (LOD) of isoalantolactone and alantolactone was 0.8 and $1 \mathrm{ng} / \mathrm{mL}$, and the lower LOQ was 2.5 and $4 \mathrm{ng} / \mathrm{mL}$ (Figure 2). 
TABLE 3: Stability of samples under different storage conditions $(n=6)$.

\begin{tabular}{|c|c|c|c|c|c|}
\hline \multirow{3}{*}{ Condition } & \multicolumn{3}{|c|}{ Concentration (ng/mL) } & \multicolumn{2}{|c|}{ RSD\% } \\
\hline & \multirow{2}{*}{ Added } & \multicolumn{2}{|c|}{ Measured } & \multirow{2}{*}{ Isoalantolactone } & \multirow{2}{*}{ Alantolactone } \\
\hline & & Isoalantolactone & Alantolactone & & \\
\hline \multirow{3}{*}{ Three freeze-thaw cycles } & 5 & 4.42 & 4.17 & 8.24 & 11.61 \\
\hline & 50 & 44.37 & 42.87 & 5.40 & 6.78 \\
\hline & 400 & 384.45 & 365.34 & 14.68 & 7.10 \\
\hline \multirow{3}{*}{ Freezing for 20 days $\left(-80^{\circ} \mathrm{C}\right)$} & 5 & 3.98 & 4.27 & 11.42 & 10.52 \\
\hline & 50 & 38.34 & 44.20 & 3.77 & 6.43 \\
\hline & 400 & 369.48 & 391.30 & 7.24 & 11.16 \\
\hline \multirow{3}{*}{ Room temperature $\left(20^{\circ} \mathrm{C}\right), 4 \mathrm{~h}$} & 5 & 4.72 & 4.10 & 9.42 & 8.50 \\
\hline & 50 & 43.23 & 41.29 & 1.44 & 5.33 \\
\hline & 400 & 373.84 & 367.14 & 14.10 & 13.77 \\
\hline \multirow{3}{*}{ Autosampler for $24 \mathrm{~h}\left(20^{\circ} \mathrm{C}\right)$} & 5 & 4.67 & 4.75 & 8.11 & 7.96 \\
\hline & 50 & 45.51 & 46.12 & 4.56 & 2.11 \\
\hline & 400 & 376.10 & 370.43 & 9.76 & 5.08 \\
\hline
\end{tabular}

The calibration curves, constructed using linear least squares regression, suggested good linearity in rat plasma. A typical calibration curve equation for isoalantolactone and alantolactone was $y=0.1205 x+0.0195\left(r^{2}=0.9991\right)$ and $y=0.0602 x-0.007\left(r^{2}=0.9987\right)$, where $y$ represents the ratio of the isoalantolactone or alantolactone peak area to the osthole peak area ( $x$ is the concentration of isoalantolactone or alantolactone in rat plasma and $r$ is about the correlation coefficient).

3.3.3. Precision and Accuracy. Precision and accuracy of this assay were determined by analysis of quality control (QC) samples in the blank rat plasma. Intraday precision was $7.06 \%$ or less and the interday precision was $9.57 \%$ or less at each QC level. The accuracy of intraday and interday performances of validation QC at concentrations of 5, 50, and $400 \mathrm{ng} / \mathrm{mL}$ is listed in Table 1 . The assay values on both the occasions (intraand interday) were within the accepted variable limits.

3.3.4. Recovery and Matrix Effect. Data on absolute recovery and matrix effect are shown in Table 2. In all samples, the average extraction recovery rates of the two lactones were obtained by analyzing six QC samples at low, medium, and high concentrations, respectively. The low concentration should be slightly above but close to LLOQ and the high concentration should be lower but close to the highest concentration on the standard curve. The matrix effect for two lactones ranged from $100.84 \%$ to $109.44 \%$ and it was $101.76 \%$ for osthole. These near $100 \%$ results suggested that the matrix exerted minimal effects on the method in response to analytes. The results were within the assay variability limits during the entire process.

3.3.5. Stability. The extracted quality control samples of the lactones were found to be stable in autosampler, room temperature $\left(20^{\circ} \mathrm{C}\right)$, freeze-thaw and long-term (20 days) storage, suggesting that the established method was suitable for the pharmacokinetic study (Table 3).
TABLE 4: Mean plasma concentrations of the lactones $(C, \mathrm{ng} / \mathrm{mL})$ over time.

\begin{tabular}{lcc}
\hline \multirow{2}{*}{$(\mathrm{h})$} & \multicolumn{2}{c}{ i.v. administration $(5 \mathrm{mg} / \mathrm{kg})$} \\
& Isoalantolactone & Alantolactone \\
\hline 0.083 & $356.69 \pm 79.93^{\mathrm{a}}$ & $336.23 \pm 75.56$ \\
0.25 & $80.31 \pm 44.97$ & $121.87 \pm 40.28$ \\
0.5 & $29.04 \pm 9.71$ & $66.01 \pm 10.94$ \\
0.75 & $16.97 \pm 7.63$ & $54.52 \pm 5.64$ \\
1 & $8.10 \pm 2.06$ & $44.10 \pm 8.65$ \\
2 & $7.44 \pm 1.05$ & $22.87 \pm 7.04$ \\
4 & $5.63 \pm 1.80$ & $14.03 \pm 2.59$ \\
6 & $4.05 \pm 0.72$ & $8.05 \pm 1.37$ \\
10 & $3.57 \pm 0.75$ & $5.92 \pm 1.16$ \\
\hline
\end{tabular}

${ }^{\mathrm{a}}$ Mean $\pm \mathrm{SD}$.

TABLE 5: Pharmacokinetic parameters of the lactones in rats ${ }^{\mathrm{a}, \mathrm{b}}$.

\begin{tabular}{lcc}
\hline \multirow{2}{*}{ Parameter } & \multicolumn{2}{c}{ i.v. administration $(5 \mathrm{mg} / \mathrm{kg})$} \\
& Isoalantolactone & Alantolactone \\
\hline$t_{1 / 2}$ Kel $(\mathrm{h})$ & $0.17 \pm 0.08^{* *}$ & $0.60 \pm 0.32$ \\
$\mathrm{MRT}(\mathrm{h})$ & $4.86 \pm 2.86$ & $4.80 \pm 2.51$ \\
$\mathrm{CL}(\mathrm{L} / \mathrm{h} / \mathrm{kg})$ & $30.61 \pm 6.16^{* *}$ & $18.59 \pm 1.54$ \\
$C_{\max }(\mathrm{ng} / \mathrm{mL})$ & $0.13 \pm 0.05^{*}$ & $0.17 \pm 0.07$ \\
AUC $(\mathrm{h} \mathrm{ng} / \mathrm{mL})$ & $170.19 \pm 41.12^{* *}$ & $270.49 \pm 22.10$ \\
AUMC $(\mathrm{h} \mathrm{ng} / \mathrm{mL})$ & $903.99 \pm 803.19$ & $1303.44 \pm 688.08$ \\
Vss $(\mathrm{L})$ & $138.44 \pm 54.14$ & $88.99 \pm 46.07$ \\
$A$ & $775.41 \pm 233.50$ & $389.92 \pm 206.83$ \\
$B$ & $764.81 \pm 231.31$ & $359.33 \pm 195.86$ \\
Alpha & $10.60 \pm 3.73$ & $30.59 \pm 13.43$ \\
Beta & $9.77 \pm 3.75$ & $4.11 \pm 2.31$ \\
\hline
\end{tabular}

${ }^{\mathrm{a}}$ Mean \pm SD.

${ }^{b}$ Statistical significance indicated by ${ }^{*} P<0.05$ and ${ }^{* *} P<0.01$ versus the alantolactone group. 


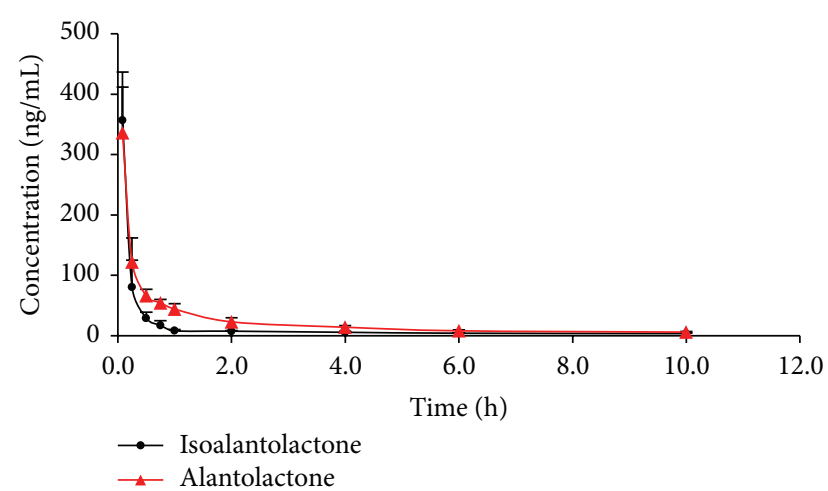

Figure 3: Mean \pm SD plasma concentration-time profiles of isoalantolactone and alantolactone in rat plasma following i.v. administration at $5 \mathrm{mg} / \mathrm{kg}$ dose, respectively.

3.3.6. Sample Analysis of the Pharmacokinetic Study. The method was applied to a pharmacokinetic study of isoalantolactone and alantolactone separately in rats. The average concentration-time profiles of six rats after intravenous injection of $5 \mathrm{mg} / \mathrm{kg}$ and its data on mean plasma concentration of the two lactones over time were given in Tables 4 and 5 . The concentration-time curves were shown in Figure 3. Our results indicated that isoalantolactone and alantolactone were distributed and eliminated rapidly possibly due to its lowpolarity characteristic and isoalantolactone was eliminated faster than alantolactone in rat plasma after intravenous injection of $5 \mathrm{mg} / \mathrm{kg}$. A large apparent volume of distribution $(138.44 \pm 54.14$ and $88.99 \pm 46.07 \mathrm{~L})$ suggests that the lactones may accumulate in certain organs with high concentration after intravenous injection. The plasma concentration profiles of the both two lactones in rats fitted to two-compartment models after administration separately. The level of $t_{1 / 2}$, Kel, $\mathrm{CL}, C_{\max }$, and AUC was significantly increased in the alantolactone group compared to isoalantolactone group. These results were so different from the previous report in which simultaneous administration was applied [15]. It seemed like the effect of drug-drug interactions in metabolism in vivo can be avoided in a separating administration study. However, further research is required to identify the effect of the two sesquiterpene lactones on drug-metabolizing enzymes.

\section{Disclosure}

Renjie Xu was the first author.

\section{Conflict of Interests}

The authors declare that there is no conflict of interests regarding the publication of this paper.

\section{Acknowledgments}

This work was supported by the Important National Science \& Technology Specific Projects funded by the Chinese Government (2012ZX09103201-038). The authors thank SJTULAB for their research support.

\section{References}

[1] F. Bohlmann, P. K. Mahanta, J. Jakupovic, R. C. Rastogi, and A. A. Natu, "New sesquiterpene lactones from Inula species," Phytochemistry, vol. 17, no. 7, pp. 1165-1172, 1978.

[2] A. Stojakowska, K. Michalska, and J. Malarz, "Simultaneous quantification of eudesmanolides and thymol derivatives from tissues of Inula helenium and I. royleana by reversedphase high-performance liquid chromatography," Phytochemical Analysis, vol. 17, no. 3, pp. 157-161, 2006.

[3] Y. Li, Z.-Y. Ni, M.-C. Zhu et al., "Antitumour activities of sesquiterpene lactones from Inula helenium and Inula japonica," Zeitschrift für Naturforschung C, vol. 67, no. 7-8, pp. 375380, 2012.

[4] J. Chun, R. J. Choi, S. Khan et al., "Alantolactone suppresses inducible nitric oxide synthase and cyclooxygenase- 2 expression by down-regulating NF- $\kappa \mathrm{B}$, MAPK and AP-1 via the MyD88 signaling pathway in LPS-activated RAW 264.7 cells," International Immunopharmacology, vol. 14, no. 4, pp. 375-383, 2012.

[5] G. Wenhua, C. Yaowen, Y. Yegao, C. Xingguo, and H. Zhide, "Separation and determination of two sesquiterpene lactones in Radix inulae and Liuwei Anxian San by microemulsion electrokinetic chromatography," Biomedical Chromatography, vol. 18, no. 10, pp. 826-832, 2004.

[6] Z. Stojanović-Radić, L. Čomić, N. Radulović et al., "Antistaphylococcal activity of Inula helenium L. root essential oil: eudesmane sesquiterpene lactones induce cell membrane damage," European Journal of Clinical Microbiology and Infectious Diseases, vol. 31, no. 6, pp. 1015-1025, 2012.

[7] X.-L. Xin, X.-C. Ma, K.-X. Liu, J. Han, B.-R. Wang, and D.A. Guo, "Microbial transformation of alantolactone by Mucor polymorphosporus," Journal of Asian Natural Products Research, vol. 10, no. 10, pp. 933-937, 2008.

[8] T. J. Schmidt, R. Brun, G. Willuhn, and S. A. Khalid, "Antitrypanosomal activity of helenalin and some structurally related sesquiterpene lactones," Planta Medica, vol. 68, no. 8, pp. 750751, 2002.

[9] J. Qiu, M. Luo, J. Wang et al., "Isoalantolactone protects against Staphylococcus aureus pneumonia," FEMS Microbiology Letters, vol. 324, no. 2, pp. 147-155, 2011.

[10] M. Khan, C. Ding, A. Rasul et al., "Isoalantolactone induces reactive oxygen species mediated apoptosis in pancreatic carcinoma PANC-1 cells," International Journal of Biological Sciences, vol. 8, no. 4, pp. 533-547, 2012.

[11] W. Wei, H. Huang, S. Zhao et al., "Alantolactone induces apoptosis in chronic myelogenous leukemia sensitive or resistant to imatinib through NF- $\kappa \mathrm{B}$ inhibition and $\mathrm{Bcr} / \mathrm{Abl}$ protein deletion," Apoptosis, vol. 18, no. 9, pp. 1060-1070, 2013.

[12] M. Khan, F. Yi, A. Rasul et al., "Alantolactone induces apoptosis in glioblastoma cells via GSH depletion, ROS generation, and mitochondrial dysfunction," IUBMB Life, vol. 64, no. 9, pp. 783794, 2012.

[13] D. Chandrapal Reddy, A. T. Bapuji, V. Rao Suryanarayana et al., "Development and validation of a LC/MS/MS method for the determination of duloxetine in human plasma and its application to pharmacokinetic study," E-Journal of Chemistry, vol. 9, no. 2, pp. 899-911, 2012.

[14] T. Yasuda, M. Tanaka, and K. Iba, "Quantitative determination of amlodipine in serum by liquid chromatography with atmospheric pressure chemical ionization tandem mass 
spectrometry," Journal of Mass Spectrometry, vol. 31, no. 8, pp. 879-884, 1996.

[15] C. Guo, S. Zhang, S. Teng, and K. Niu, "Simultaneous determination of sesquiterpene lactones isoalantolactone and alantolactone isomers in rat plasma by liquid chromatography with tandem mass spectrometry: application to a pharmacokinetic study," Journal of Separation Science, vol. 37, no. 8, pp. 950-956, 2014.

[16] J. Y. Seo, S. S. Lim, J. R. Kim et al., "Nrf2-mediated induction of detoxifying enzymes by alantolactone present in inula helenium," Phytotherapy Research, vol. 22, no. 11, pp. 1500-1505, 2008.

[17] M. Liu, H. Liu, X. Lu, C. Li, Z. Xiong, and F. Li, "Simultaneous determination of icariin, icariside II and osthole in rat plasma after oral administration of the extract of Gushudan (a Chinese compound formulation) by LC-MS/MS," Journal of Chromatography B: Analytical Technologies in the Biomedical and Life Sciences, vol. 860, no. 1, pp. 113-120, 2007.

[18] J. Zeng, Biomedical Analysis, Peking University Medical Press, Beijing, China, 2nd edition, 1998. 

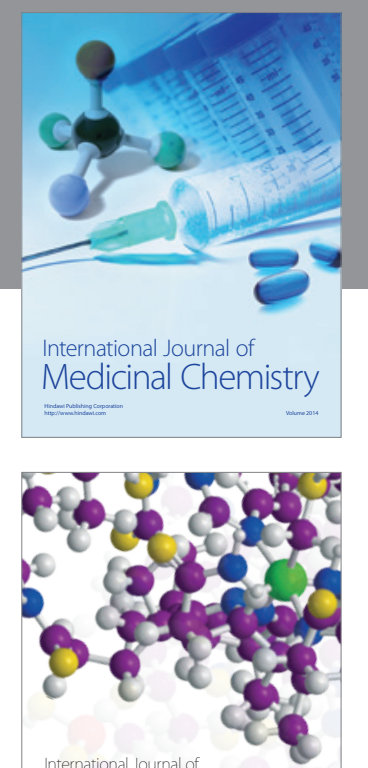

\section{Carbohydrate} Chemistry

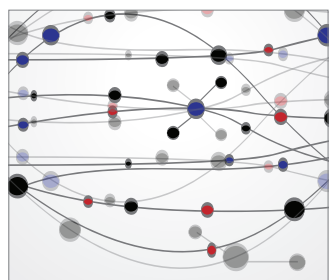

The Scientific World Journal
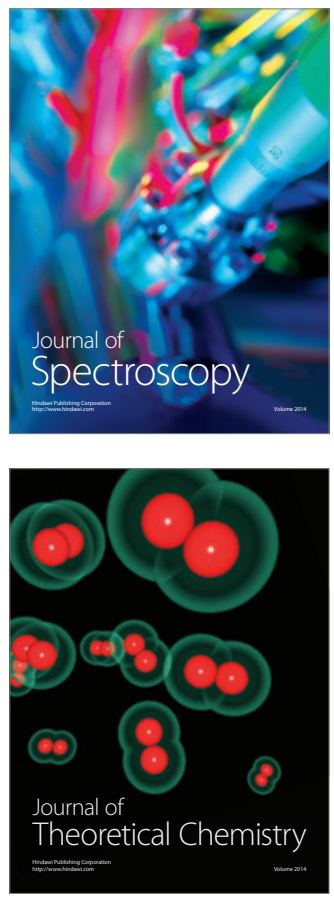
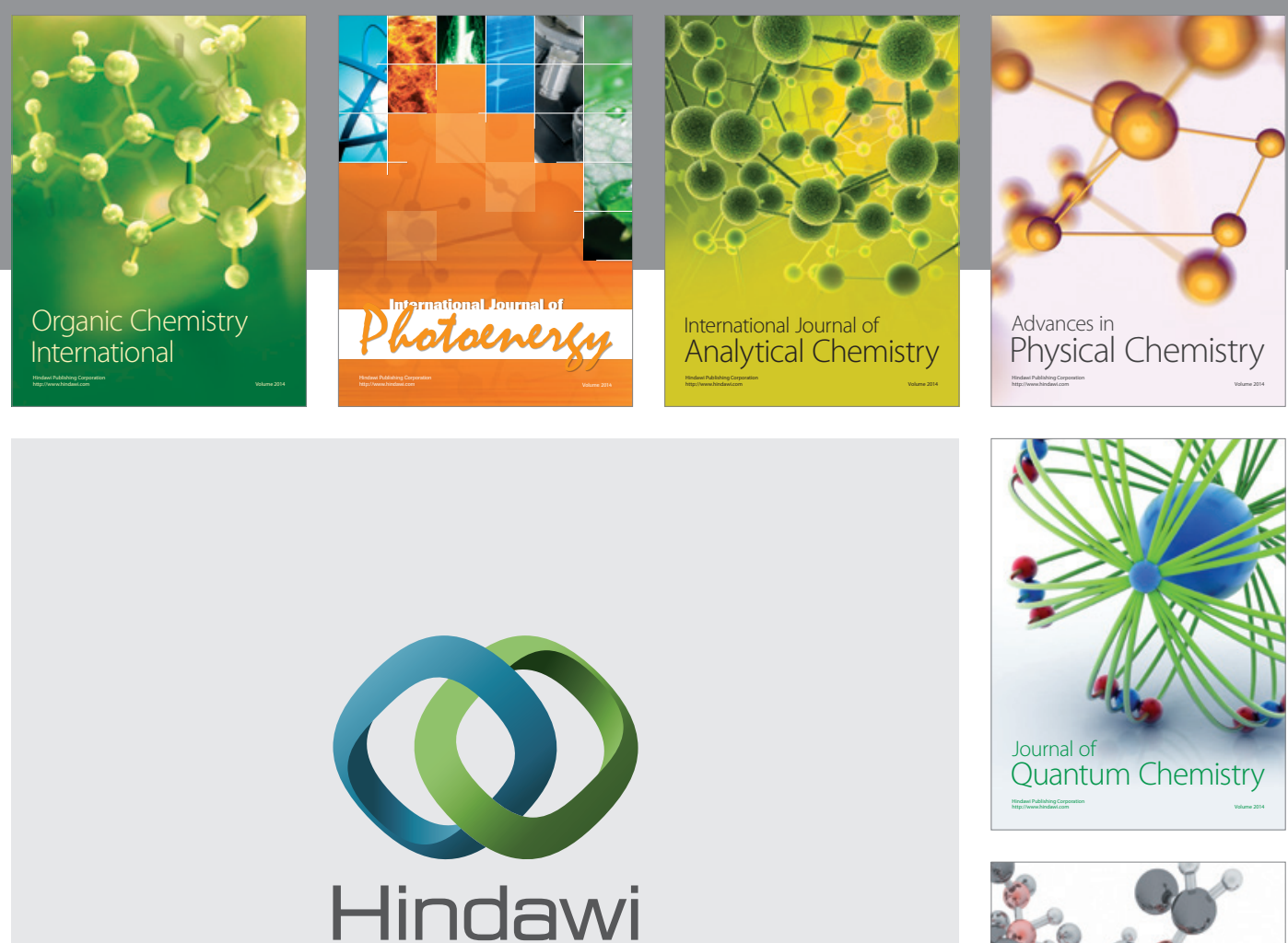

Submit your manuscripts at

http://www.hindawi.com

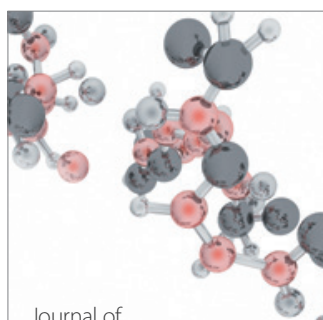

Analytical Methods

in Chemistry

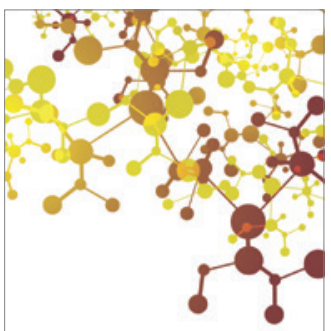

Journal of

Applied Chemistry

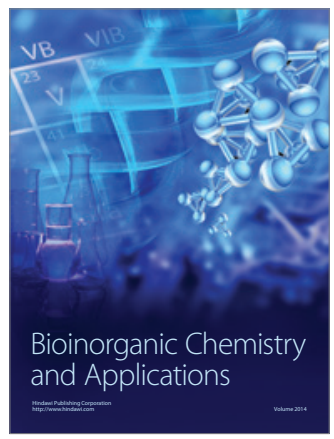

Inorganic Chemistry
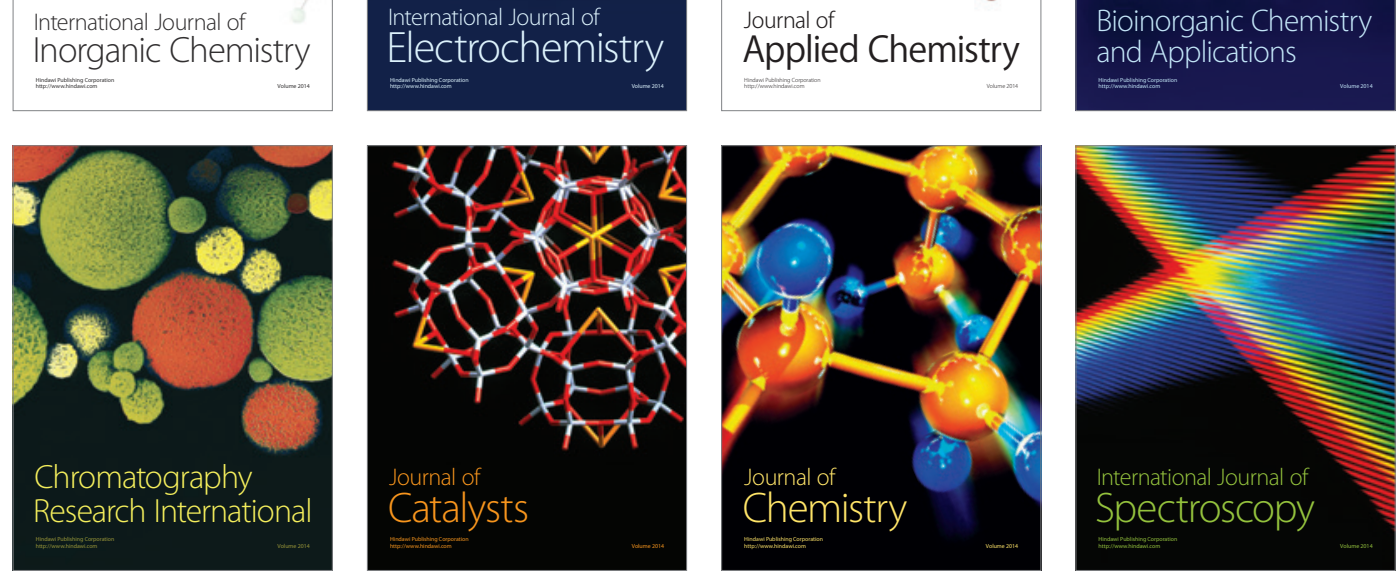